\title{
Novel solid-state-detector dedicated cardiac camera for fast myocardial perfusion imaging: multicenter comparison with standard dual detector cameras
}

\author{
Fabio P. Esteves, MD, ${ }^{\text {a }}$ Paolo Raggi, MD, PhD, ${ }^{a}$ Russell D. Folks, CNMT, ${ }^{a}$ \\ Zohar Keidar, MD, PhD, ${ }^{b}$ J. Wells Askew, MD, ${ }^{c}$ Shmuel Rispler, MD, PhD, ${ }^{b}$ \\ Michael K. O'Connor, PhD, ${ }^{\mathrm{C}}$ Liudmilla Verdes, MD, ${ }^{\mathrm{a}}$ and Ernest V. Garcia, $\mathrm{PhD}^{\mathrm{a}}$
}

Objective. To compare the diagnostic performance of a new dedicated ultrafast solid-state cardiac camera (Discovery NM 530c [DNM]) with standard dual detector cameras (S-SPECT) in myocardial perfusion imaging. The primary goal was a per-patient analysis of diagnostic performance of the DNM using S-SPECT as the reference standard.

Methods and results. In total, 168 patients underwent one-day Tc-99m tetrofosmin rest/ stress myocardial perfusion SPECT. DNM and S-SPECT images were obtained with the same injected doses. The DNM camera uses an array of cadmium zinc telluride pixilated detectors and a multipinhole collimator simultaneously imaging all cardiac views with no moving parts. Rest and stress acquisition times were 4 and 2 minutes for DNM and 14 and 12 minutes for S-SPECT. Two blinded readers independently interpreted all scans on a patient level and on a vascular territory level using a standard five-point scale. Interobserver differences were resolved by a third observer. Agreement between DNM and S-SPECT for presence or absence of myocardial perfusion defects on a per-patient analysis was $91.9 \%$ and $92.5 \%$, respectively. Correlation coefficients of rest and stress left ventricular ejection fractions were $0.87(P<.01)$ and $0.90(P<.01)$.

Conclusion. The diagnostic performance of DNM is comparable to that of S-SPECT on a per-patient basis. However, superior image quality can be achieved with significantly shorter acquisition times with DNM because of improved count sensitivity and image contrast over S-SPECT. (J Nucl Cardiol 2009;16:927-34.)

Key Words: Instrumentation $\cdot$ SPECT $\cdot$ myocardial perfusion imaging $\cdot$ coronary artery disease $\cdot$ gated SPECT $\cdot$ tetrofosmin $\cdot$ CZT detectors

\section{INTRODUCTION}

Myocardial perfusion single photon emission computed tomography (SPECT) has been successfully implemented for the diagnosis of coronary artery disease (CAD) and risk stratification of patients with CAD.

From the Department of Radiology, ${ }^{\text {a }}$ Emory University School of Medicine, Atlanta, GA; Rambam Health Care Campus, ${ }^{\text {b }}$ Haifa, Israel; and Mayo Clinic, ${ }^{\mathrm{c}}$ Rochester, MN.

Received for publication Jun 14, 2009; final revision accepted Aug 2, 2009.

Reprint requests: Fabio P. Esteves, MD, Department of Radiology, Emory University School of Medicine, 1364 Clifton Road, NE, Atlanta, GA 30322; festeve@emory.edu.

$1071-3581 / \$ 34.00$

Copyright (C) 2009 The Author(s). This article is published with open access at Springerlink.com.

doi:10.1007/s12350-009-9137-2
Standard dual-detector SPECT (S-SPECT) cameras are used in most nuclear cardiology laboratories. Imaging time is reduced by half when compared to singledetector SPECT cameras. Iterative reconstruction algorithms using resolution recovery allow further reduction of imaging time without compromising image quality. ${ }^{1,2}$ However, these cameras use sodium iodide ( $\mathrm{NaI}[\mathrm{Tl}]$ ) detectors with parallel hole collimators and the diagnostic quality of myocardial perfusion SPECT images is limited by photon sensitivity, prolonged imaging times and artifacts enhanced by a moving detector system.

In an effort to overcome these limitations of S-SPECT, a novel dedicated ultra-fast, solid-state cardiac camera (Discovery NM 530c [DNM], GE Healthcare, Haifa, Israel) has been developed. This camera provides a 3 - to 5-fold increase in photon sensitivity (depending on 
heart size and background activity), a 1.65-fold improvement in energy resolution, and a 1.7- to 2.5-fold improvement in spatial resolution when compared to S-SPECT. ${ }^{3}$ This article describes the first validation study comparing DNM and S-SPECT for ECG-gated rest/stress Tc-99m tetrofosmin myocardial perfusion imaging.

\section{METHODS}

\section{Study Design}

This was a multicenter trial designed to compare myocardial perfusion SPECT using DNM cameras with S-SPECT cameras. The primary hypothesis of this study was to demonstrate that the per-patient diagnostic performance of the DNM (using reduced acquisition times) in detecting the presence or absence of perfusion defects was comparable (i.e. not inferior) to that of S-SPECT using well-accepted conventional imaging protocols. ${ }^{4}$ To test this hypothesis the strength of agreement between the DNM and the S-SPECT images was compared to the strength of agreement between the same S-SPECT images interpreted a second time $\left(\mathrm{S}_{3} \mathrm{SPECT}_{2}\right)$ 3 months after the original interpretation $\left(\mathrm{S}-\mathrm{SPECT}_{1}\right)$. We hypothesized that the DNM-S-SPECT ${ }_{1}$ agreement rate would not be inferior to the S-SPECT 2 -S-SPECT $T_{1}$ agreement rate. The secondary goals included the image quality, the interobserver agreement rates, the diagnostic performance of DNM based on a per-vessel analysis using S-SPECT ${ }_{1}$ as the reference standard, and the correlation of rest and stress left ventricular volumes and ejection fractions (LVEF).

\section{Study Population}

The study population consisted of 168 patients referred for myocardial perfusion SPECT for known or suspected CAD in three nuclear laboratories: 64 of the patients were from Rambam Health Care Campus in Haifa, Israel, 49 from Mayo Clinic in Rochester, Minnesota, and 55 from Emory University in Atlanta, Georgia. All three sites obtained approval from their Institutional Review Boards to perform this trial and each patient signed an informed consent form. Patients with contraindication for the procedure according to clinical guidelines and local regulations were excluded.

\section{Exercise and Pharmacologic Stress Testing}

All patients underwent one-day low-dose rest, high-dose stress Tc-99m tetrofosmin myocardial perfusion SPECT. Reststress doses were determined based on patient's body weight: $<200$ lbs (370 MBq rest [10 $\mathrm{mCi}$ ], $1110 \mathrm{MBq}$ stress [30 $\mathrm{mCi}$ ); 200-300 lbs (444 MBq rest [12 mCi], $1332 \mathrm{MBq}$ stress [36 $\mathrm{mCi}$ ); $>300$ lbs (555 MBq rest [15 mCi], $1665 \mathrm{MBq}$ stress [45 $\mathrm{mCi}]$ ). Stress testing was started a minimum of 2 hours after rest injection. Patients who underwent exercise stress testing followed the Bruce protocol. Tc-99m tetrofosmin was injected at peak stress only if patients attained $\geq 85 \%$ maximum predicted heart rate. The maximum exercise level achieved was maintained for 1 minute after tracer injection. Patients who were unable to exercise (or reach their target heart rate) and had refrained from caffeine-containing products for 12 hours prior to the exam received a 4- or 6-minute infusion of adenosine $(140 \mu \mathrm{g} / \mathrm{kg} /$ minute $)$ or a 4 -minute infusion of dipyridamole $(140 \mu \mathrm{g} / \mathrm{kg} / \mathrm{minute})$ and Tc-99m tetrofosmin was administered halfway through the adenosine infusion or 3 minutes after completion of dipyridamole infusion.

\section{Standard Dual-Detector SPECT}

All patients underwent rest and stress 8-frame ECG-gated myocardial perfusion SPECT according to the American Society of Nuclear Cardiology guidelines. ${ }^{4}$ Dual-detector cameras were used for image acquisition (CardioMD, Philips Medical Systems, Cleveland, OH; Ventri and Millennium VG, GE Healthcare, Milwaukee, WI). S-SPECT rest imaging was started immediately after DNM rest imaging at $60 \pm 15$ minutes post tracer injection. S-SPECT stress imaging was started immediately after DNM stress imaging at $30 \pm 15$ minutes post exercise stress testing and $60 \pm 15$ minutes post pharmacologic stress testing. Acquisition times were 14 minutes for rest imaging and 12 minutes for stress imaging (Figure 1).

\section{Ultra-Fast Solid-State Cardiac Camera}

The DNM is equipped with a multiple pinhole collimator and 19 stationary cadmium zinc telluride (CZT) detectors simultaneously imaging 19 cardiac views. Each detector contains $32 \times 32$ pixilated 5 -mm-thick $(2.46 \times 2.46 \mathrm{~mm}) \mathrm{CZT}$ elements. The pinhole collimation uses a $5.1 \mathrm{~mm}$ effective diameter aperture made of tungsten. System design enables high quality imaging of a three-dimensional volume imaged by all detectors (quality field-of-view), where the patient's heart is to be positioned. Patients were imaged in the supine position with arms placed over the head. Automated heart positioning in the quality field-of-view was assisted by using real-time persistence imaging. Images were acquired in list mode. Rest and stress acquisition times were 4 and 2 minutes, respectively. Although the DNM detector could be rotated by the gantry if required for positioning, once acquisition was started there is no detector or collimator motion of any kind. Maximum penalized likelihood expectation maximization iterative

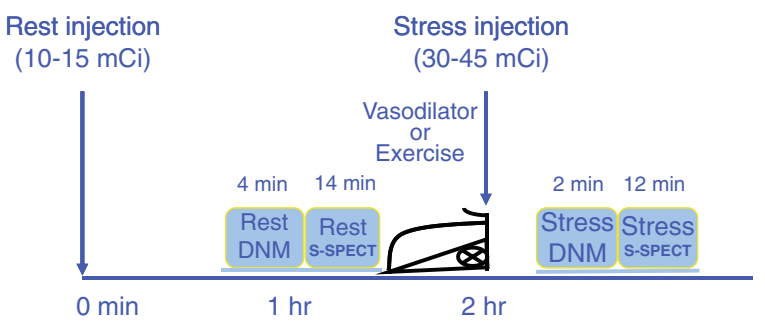

Figure 1. Schematic depiction of the rest/stress Tc-99m tetrofosmin myocardial perfusion imaging protocol. Standard dual-detector SPECT (S-SPECT) and DNM were performed with the same injected doses. Rest and stress DNM images were obtained immediately prior to S-SPECT images. 
reconstruction adapted to the DNM three-dimensional geometry was used to create transaxial slices of the left ventricle. The tomographic DNM studies were also reprojected into 60 planar projections to emulate S-SPECT layout. No corrections for scatter or attenuation were performed for either the DNM or S-SPECT system.

\section{SPECT Image Analysis}

Images were transferred to the core laboratory at Emory University via file transfer protocols. DNM and S-SPECT images were processed and visually interpreted using the Emory Cardiac Toolbox (ECTb) software version 3.1 (Syntermed, Inc, Atlanta, GA). Software-provided normal files and perfusion quantitative tools were used for the interpretation of $\mathrm{S} \mathrm{SPECT}_{1}$ and $\mathrm{S}_{\mathrm{SPECT}}$ images. A preliminary normal database was also generated for the DNM studies consisting of 20 patients with low likelihood of CAD. The same technologist performed image processing and display of all studies blinded to clinical data. The readers had a consensus meeting to evaluate 30 initial DNM imaging studies not included in the analysis prior to beginning the multicenter study. Two expert readers (FE, PR) independently interpreted all images blinded to clinical data except for patient's gender, height, and weight. The readers were also blinded as to whether the study was acquired via DNM or S-SPECT. Interobserver differences were resolved by a third observer (EG). All readers had a minimum 10 years of nuclear cardiology image interpretation experience. Image quality at rest and stress was classified as excellent, adequate, or suboptimal. The gated images were used to determine segmental wall thickening, left ventricular volumes and ejection fraction and served as adjunct to interpretation of the tomographic images. All studies were graded using a five-point scale on a subject level and on a vascular territory level (definitely normal, probably normal, equivocal, probably abnormal, or definitely abnormal). Image interpretation of MPI studies was performed using exactly the same procedure used in clinical interpretation. The diagnostician uses ECTb to view rotating planar projections, rest and stress tomographic slices, animated gated displays, functional parameters, rest/stress polar maps using database quantification to highlight both hypoperfused and reversible segments and computer generated rest/stress 17 segment scores which are confirmed or modified. The diagnostician then integrates all of this available information to score the interpretation using the five-point scale as described above. This scale was used to score whether the patient images exhibited the presence of $\mathrm{CAD}$, and if so, to localize it to one or more of the three major vascular territories (LAD, LCX, and/or RCA). Scores three or greater (i.e. equivocal, probably abnormal, or definitely abnormal) were considered positive for disease. Stress perfusion defects were defined as reversible, fixed, or mixed.

\section{Statistical Analysis}

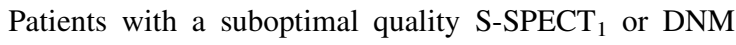
study were excluded from the primary analysis. All studies were classified as normal (definitely normal or probably normal) or abnormal (equivocal, probably abnormal, or definitely abnormal) based on the presence of one or more stress perfusion defects. The difference between two population proportions from a single sample ${ }^{5}$ was used to test if there were differences in interpretation agreement between DNM-S$\mathrm{SPECT}_{1}$ to $\mathrm{S}-\mathrm{SPECT}_{2}-\mathrm{S}-\mathrm{SPECT}_{1}$. If DNM images are equivalent to S-SPECT images, the expected difference between DNM-S-SPECT 1 agreement to S-SPECT $2-\mathrm{S}_{2} \mathrm{SPECT}_{1}$ agreement is zero. The primary analysis tested the null hypothesis of equivalence of DNM-S-SPECT $T_{1}$ agreement to S-SPECT $\mathrm{S}_{2}-\mathrm{S}$ $\mathrm{SPECT}_{1}$ agreement (no agreement rate reduction) versus inferiority (a reduction of $>0 \%$ ). A $95 \%$ confidence interval (CI) for the difference between DNM-S-SPECT 1 agreement rate to S-SPECT $T_{2}-S_{-S P C T}$ agreement rate was calculated and the null hypothesis rejected if the upper limit was below $0 \%$ with a corresponding one-tail $P$ value less than .05 .

Continuous data are presented as mean \pm standard deviation. Categorical variables are expressed in simple proportions. Image quality was compared by testing the difference between two proportions. Interobserver agreement was measured using percent agreement and kappa value. The McNemar test was used to express the differences between DNM-S-SPECT ${ }_{1}$ agreement rates to $\mathrm{S}-\mathrm{SPECT}_{2}-\mathrm{S}_{-} \mathrm{SPECT}_{1}$ agreement rates on a per-vessel analysis. The Spearman's correlation coefficient was used to compare rest and stress left ventricular end-systolic and end-diastolic volumes and LVEF. Statistical significance of the DNM-S-SPECT $_{1}$ difference in measuring LV functional parameters was determined using two-tailed paired $t$-tests assuming equal variances. A $p<.05$ was considered significant for all comparisons.

\section{RESULTS}

Seven patients were excluded from the primary analysis: four had truncation artifacts on DNM, one had an acquisition error on S-SPECT, and two underwent regadenoson stress, a protocol violation. The baseline data of the remaining 161 patients are summarized in Table 1 . Ninety patients $(56 \%)$ underwent exercise stress testing and 71 patients $(44 \%)$ pharmacologic stress testing. Only three of the seven patients excluded for the primary analysis were excluded for analysis of image quality (the two protocol violations and one of the truncated cases due to obvious image artifacts identified by the acquiring site).

\section{Primary Analysis}

Stress perfusion defects were present in 38 of 161 $(24 \%)$ studies as determined by $\mathrm{S}-S P E C T_{1}$ interpretation. There were no equivocal studies on consensus interpretation. The interobserver (FE, PR) agreement rate for DNM images on a per-patient analysis was $89 \%$ $(\mathrm{kappa}=.70,95 \% \mathrm{CI}: .57$ to .83$)$ compared to $94 \%$ for $\mathrm{S}_{-S_{P}} \mathrm{~T}_{1}$ images (kappa $=.79,95 \% \mathrm{CI}: .68$ to .90$)$. The consensus agreement rates for the absence or 
Table 1. Characteristics of the study population

\begin{tabular}{ll}
\hline Sample size & 161 \\
Age (years) & $62 \pm 12$ \\
Male gender & $66 \%(107)$ \\
Body mass index $\left(\mathrm{kg} / \mathrm{m}^{2}\right)$ & $29.1 \pm 4.9$ \\
Hypertension & $70 \%(113)$ \\
Hyperlipidemia & $75 \%(121)$ \\
Diabetes mellitus & $29 \%(48)$ \\
Smoking history & $22 \%(36)$ \\
Family history & $33 \%(53)$ \\
Prior myocardial infarction & $21 \%(34)$ \\
Prior revascularization & $42 \%(68)$ \\
Chest pain & $40 \%(65)$ \\
\hline
\end{tabular}

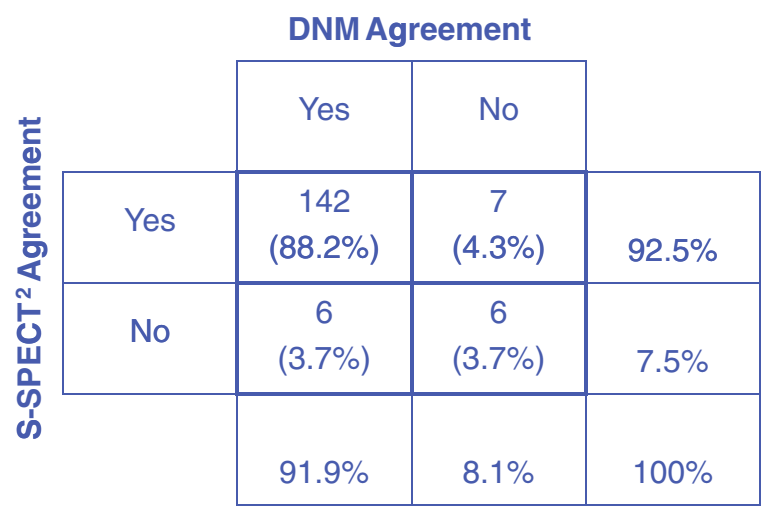

Figure 2. Consensus agreement rates for the absence or presence of stress perfusion defects of DNM and S-SPECT images using S-SPECT $T_{1}$ images as the reference standard.

presence of stress perfusion defects of DNM and $\mathrm{S}_{-} \mathrm{SPECT}_{2}$ images are summarized in Figure 2. The overall agreement rate was $91.9 \%$ for DNM-S-SPECT ${ }_{1}$ and was $92.5 \%$ for $\mathrm{S}_{-S^{2}} \mathrm{SECT}_{2}-\mathrm{S}_{-} \mathrm{SPECT}_{1}(P=.99)$. Thus, interpretation agreement difference between DNM and $\mathrm{S}-\mathrm{SPECT}_{2}$ was $-0.6 \%$ (95\% CI: $-5.0 \%$ to $3.8 \%$ ). The upper limit of the $95 \% \mathrm{CI}$ is above $0 \%(P=.39)$ showing that DNM is comparable (i.e. not inferior) to
S-SPECT cameras for determining the absence or presence of stress myocardial perfusion defects.

\section{Secondary Analyses}

There were 330 rest and 330 stress images that could be evaluated for quality (165 rest/stress studies for each reader). Combining the results of the two readers, DNM rest images were considered excellent in $83 \%$ of cases compared to $70 \%$ of S-SPECT rest images $(P<.001)$. DNM stress images were considered excellent in $86 \%$ of cases compared to $68 \%$ of S-SPECT stress images $(P<.001)$ (Table 2$)$. Four patients with large body habitus (body mass index $[\mathrm{BMI}] \geq 40 \mathrm{~kg} / \mathrm{m}^{2}$ ) had severe truncation artifacts on DNM images because they were not positioned in the quality field-of-view.

Figure 3 depicts S-SPECT and DNM images in a male patient with reversible defects in the left anterior descending coronary artery and right coronary artery territories.

The DNM-S-SPECT $T_{1}$ agreement rates for the absence or presence of stress perfusion defects on a pervessel basis are shown in Figure 4. The overall agreement rate (defect present or absent) was $96 \%$ in left anterior descending artery (95\% CI: 93-99\%) and in left circumflex artery territories (95\% CI: 93-99\%). The agreement rate for the absence or presence of perfusion defects in the distribution of the right coronary artery was 93\% (95\% CI: 89-97\%). No significant differences were observed between DNM-S-SPECT ${ }_{1}$ and S-SPECT ${ }_{2}$-S$\mathrm{SPECT}_{1}$ agreement rates on a per-vessel analysis.

The correlation of rest and stress left ventricular volumes and ejection fractions between DNM and S-SPECT is depicted in Figure 5. Left ventricular ejection fractions and volumes were significantly correlated $(P<.01$ for all $)$. Correlation coefficients $(r)$ were as follows: rest left ventricular ejection fraction 0.87 (95\% CI: 0.83-0.90), rest end-systolic volume 0.96 (95\% CI: 0.94-0.97), rest end-diastolic volume 0.94 (95\% CI: 0.92-0.96), stress left ventricular ejection fraction 0.90 (95\% CI: $0.87-0.93$ ), stress end-systolic volume 0.97 (95\% CI: $0.97-0.98)$, stress end-diastolic volume 0.96

Table 2. Image quality by standard dual-detector SPECT (S-SPECT) cameras and by the DNM camera

\begin{tabular}{lcccc}
\hline & $\begin{array}{c}\text { Rest S-SPECT } \\
(\mathbf{n}=\mathbf{3 3 0})\end{array}$ & $\begin{array}{c}\text { Rest DNM } \\
(\mathbf{n}=\mathbf{3 3 0})\end{array}$ & $\begin{array}{c}\text { Stress S-SPECT } \\
(\mathbf{n}=\mathbf{3 3 0})\end{array}$ & $\begin{array}{c}\text { Stress DNM } \\
(\mathbf{n}=330)\end{array}$ \\
\hline Excellent & $232(70.3 \%)^{*}$ & $275(83.3 \%)^{*}$ & $224(67.9 \%)^{* *}$ & $282(85.5 \%)^{* *}$ \\
Adequate & $94(28.5 \%)^{*}$ & $47(14.2 \%)^{*}$ & $105(31.8 \%)^{*}$ & $41(12.4 \%)^{*}$ \\
Suboptimal & $4(1.2 \%)$ & $8(2.4 \%)$ & $1(0.3 \%)$ & $7(2.1 \%)$ \\
\hline
\end{tabular}

There were no significant differences in the rate of suboptimal quality studies at rest or stress between S-SPECT and DNM. For all other comparisons $\left({ }^{*},{ }^{*} *, \boldsymbol{\uparrow}, \uparrow\right), P<.05$. 
A

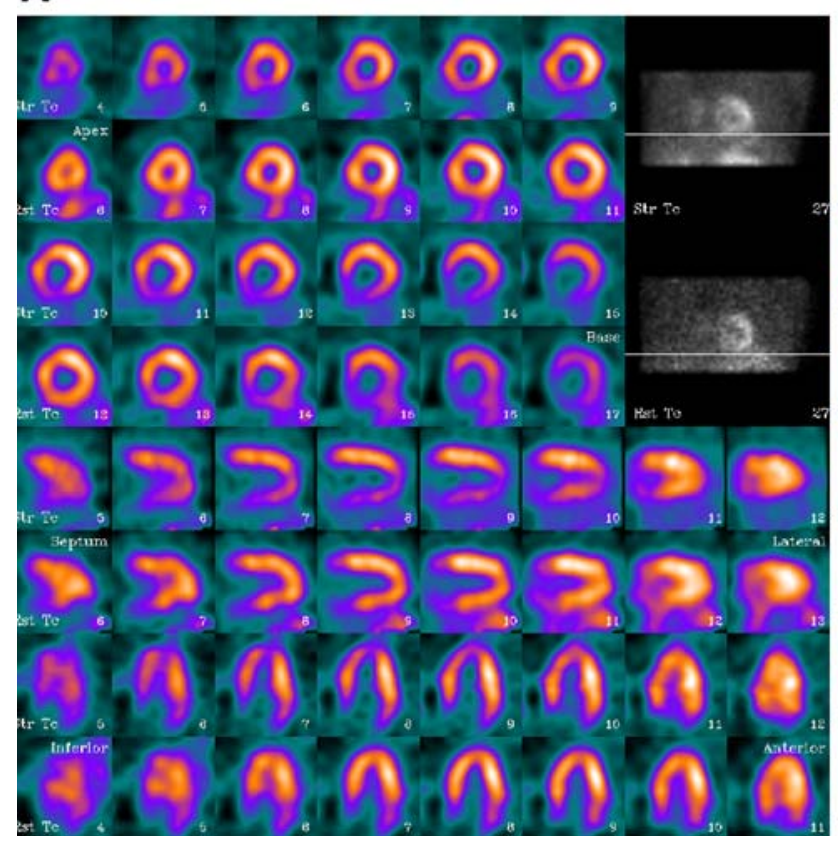

B

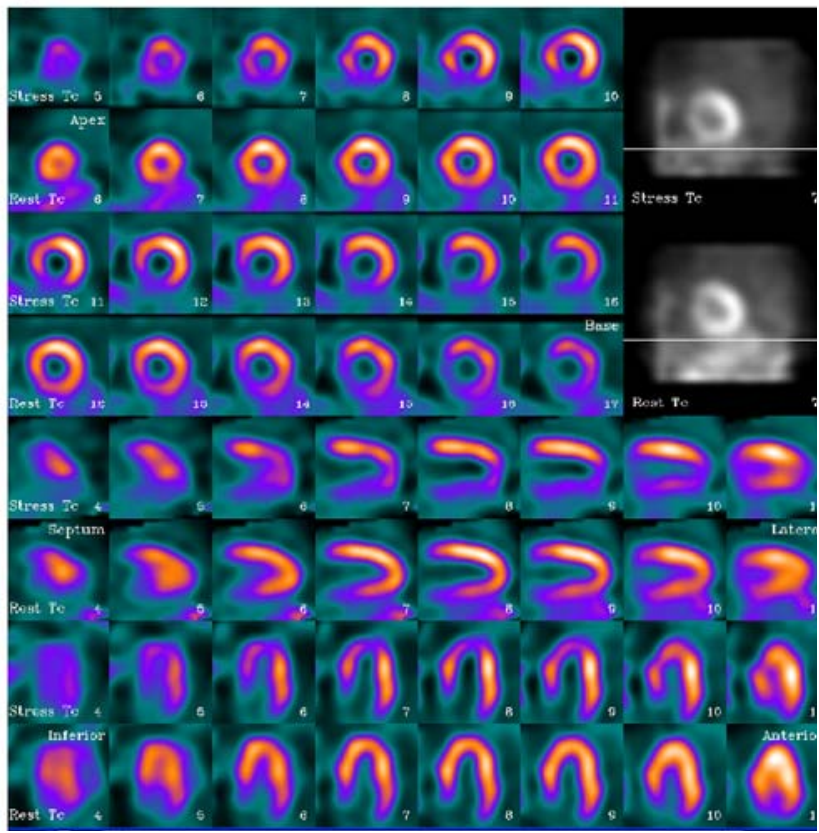

Figure 3. Standard dual-detector SPECT (S-SPECT) (A) and DNM (B) images demonstrate reversible perfusion defects of the anterior-apical and inferior walls in a 57-year-old male. Note the improved contrast and resolution of DNM over S-SPECT.

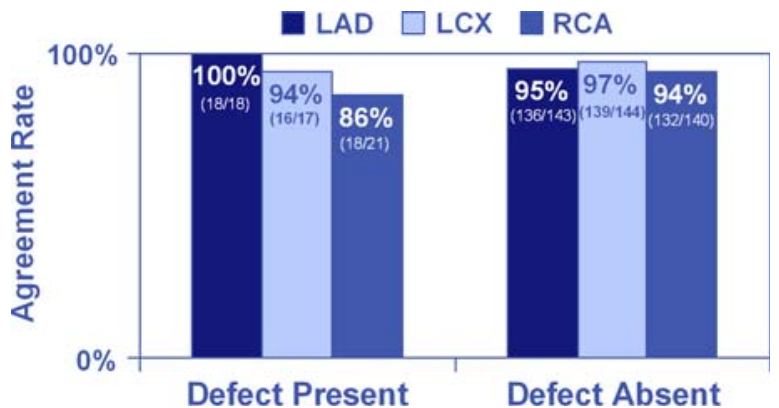

Figure 4. Consensus agreement rates between DNM-S$\mathrm{SPECT}^{1}$ images on a per-vessel analysis. There were no significant differences when compared to $\mathrm{SPECT}_{2}-\mathrm{S}_{-} \mathrm{SPECT}_{1}$ agreement rates. $L A D$, Left anterior descending artery; $L C X$, left circumflex artery; $R C A$, right coronary artery.

(95\% CI: 0.95-0.97). There was a slight trend toward higher ejection fractions on S-SPECT over DNM for both rest and stress measurements. Figure 6 shows the comparison of the two methods by means of BlandAltman plots.

\section{DISCUSSION}

In this multicenter study we demonstrated that the imaging accuracy of the new solid state, high speed, cadmium zinc camera is comparable to that of stateof-the-art dual detector conventional SPECT for detection of the presence or absence of hypoperfused vascular territories and for measurements of parameters of left ventricular function. Furthermore, because of its increased photon sensitivity and contrast resolution, this solid-state camera exhibited improved image quality over conventional SPECT even when using significantly shorter acquisition times.

Multipinhole SPECT with $\mathrm{NaI}(\mathrm{Tl})$ crystals has been shown to improve spatial resolution and detection efficiency in small animal imaging when compared to parallel-hole collimator rotational SPECT. ${ }^{6-8}$ Steele et $\mathrm{al}^{9}$ have demonstrated comparable clinical results between three $\mathrm{NaI}(\mathrm{Tl})$ detector 18-pinhole SPECT and conventional rotational SPECT. The multipinhole collimation design of the DNM camera allows the simultaneous acquisition of all views without the need for motion of the detectors. This capability could potentially reduce quality control procedures, camera downtime and service costs. In addition, inconsistencies of multiple projection datasets are decreased compared to S-SPECT because all detectors are active during the entire acquisition time. As a result there is optimal angular data collection for increased efficiency of iterative reconstruction methods. CZT-based multipinhole detectors have been demonstrated to further enhance the performance of myocardial perfusion imaging. ${ }^{10}$

Preliminary clinical validations have also been performed using a CZT-based system different from the one 
A

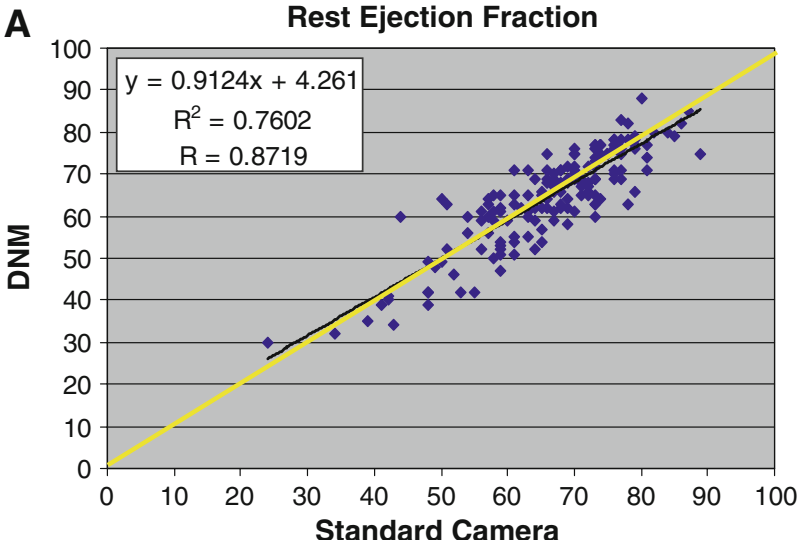

C

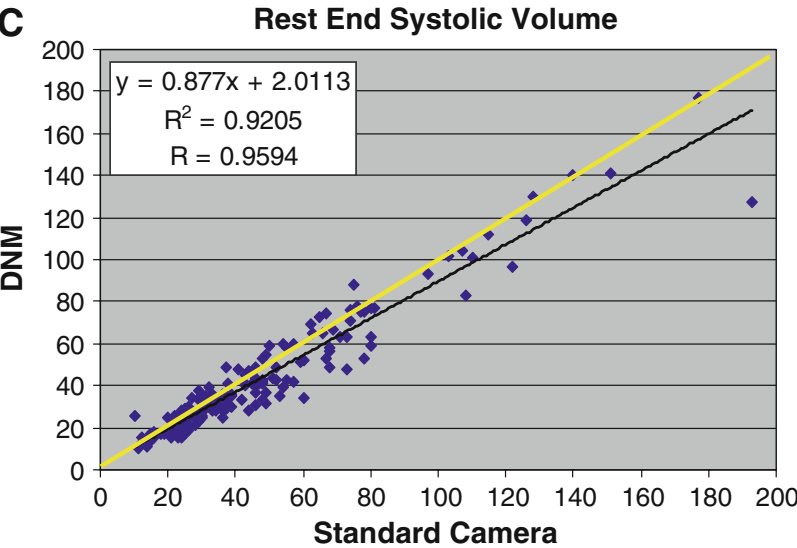

E

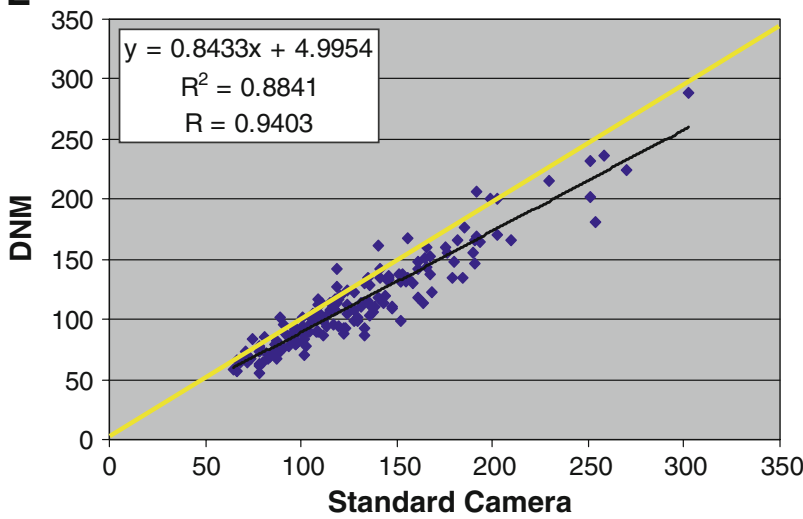

B

Stress Ejection Fraction

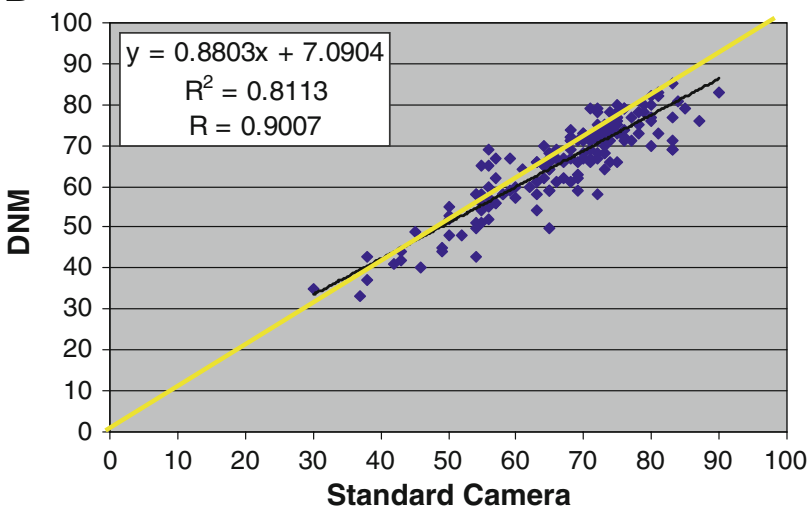

D

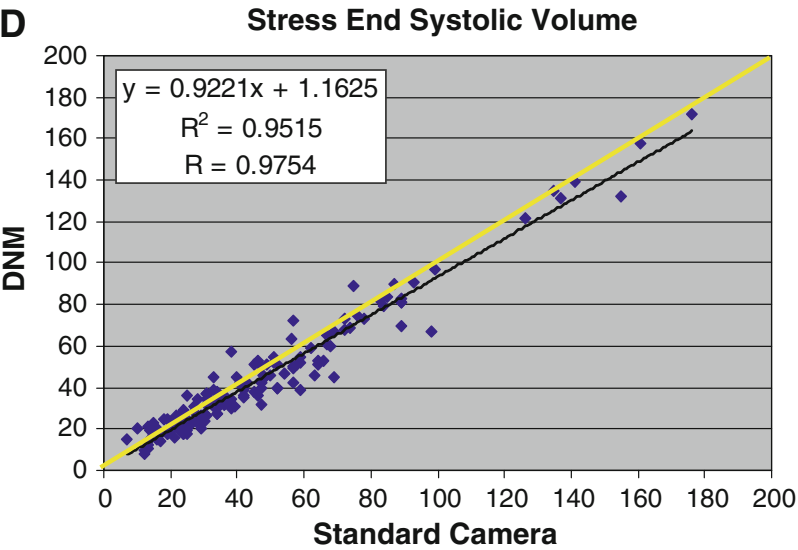

$\mathbf{F}$

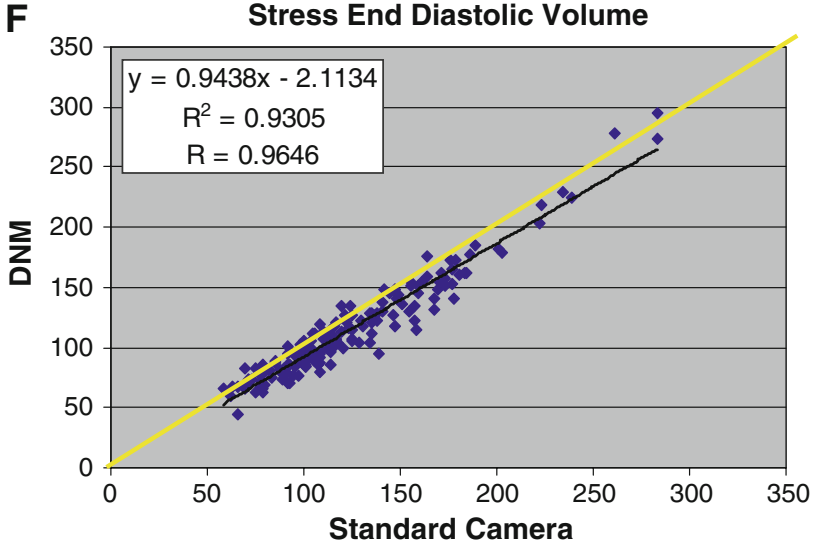

Figure 5. Correlation of left ventricular rest and stress ejection fractions (A, B), end-systolic volumes $(\mathbf{C}, \mathbf{D})$, and end-diastolic volumes $(\mathbf{E}, \mathbf{F})$ between 8-frame DNM and S-SPECT images. The dark line represents the line of regression, the yellow line the line of identity.

reported here. This other SPECT device (D-SPECT, Spectrum Dynamics, Caesarea, Israel) is equipped with nine vertical columns of CZT detectors that rotate in synchrony and are placed in a $90^{\circ}$ geometry. Sharir et al ${ }^{11}$ used this device in 44 patients and showed excellent linear correlation between summed rest and stress scores when compared with conventional SPECT. Gambhir et $\mathrm{al}^{12}$ used the same device as Sharir et $\mathrm{al}^{11}$ and reported qualitatively better myocardial edge definition in all 18 patients studied with the D-SPECT camera compared to conventional SPECT. Left ventricular ejection fraction, end-systolic and end-diastolic volumes were favorably compared between D-SPECT and conventional SPECT in two patients. Berman et al ${ }^{13}$ using the same D-SPECT camera demonstrated that a fast sequential stress thallium rest Tc-99m dual isotope protocol could be implemented 

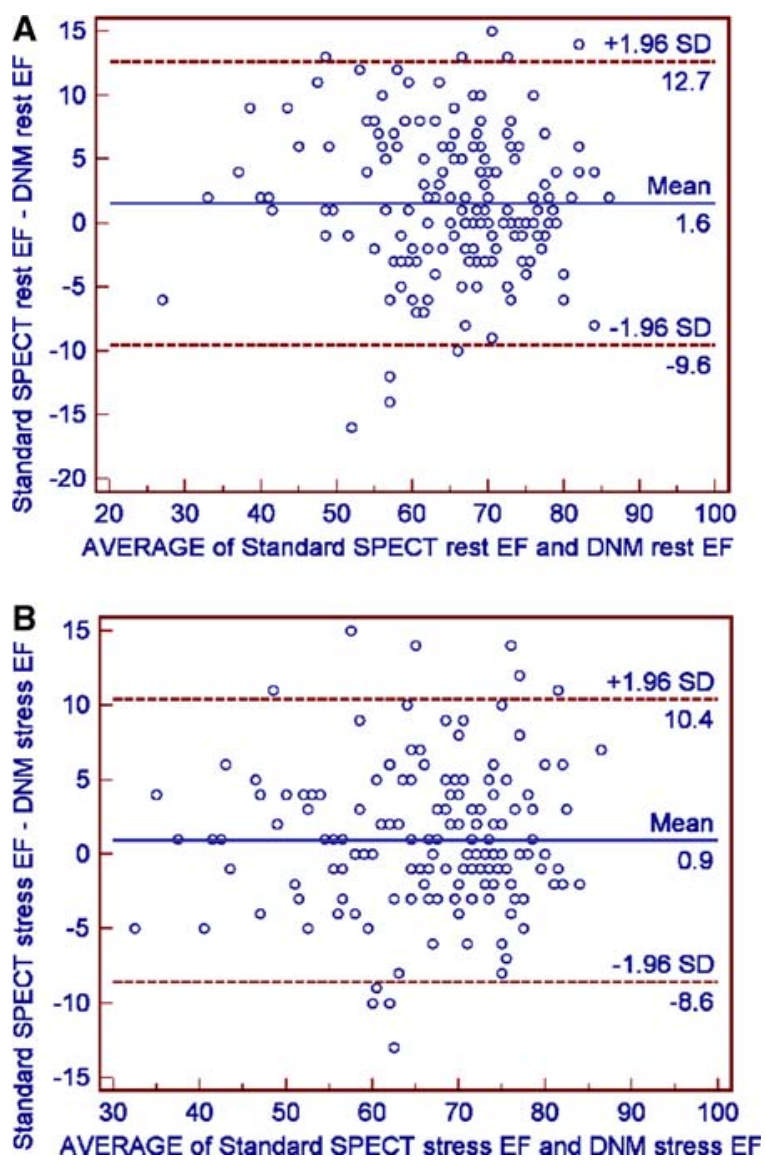

Figure 6. Bland-Altman plots depict the agreement between standard SPECT and DNM left ventricular ejection fractions (EF): rest (A) and stress (B).

with similar image quality and dosimetry as that of a rest/ stress Tc-99m protocol. In this study the investigators compared the myocardial perfusion interpretation of two large but distinct groups; 374 patients undergoing a stress Tl-201/rest Tc-99m protocol versus 262 patients undergoing rest/stress Tc- $99 \mathrm{~m} / \mathrm{Tc}-99 \mathrm{~m}$ protocol. Both groups were imaged with the D-SPECT device. These investigators noted that a limitation of their work was the lack of validation of their perfusion and function findings.

Advances in reconstruction software have also been incorporated into the DNM system. A proprietary, fully three-dimensional iterative reconstruction algorithm takes into account acquisition physics and geometry. Reconstruction parameters were optimized to achieve improved image resolution in combination with better noise control as compared to standard reconstruction. ${ }^{14}$ The DNM system has been shown to provide a 1.65-fold improvement in energy resolution, a 1.7- to 2.5-fold improvement in spatial resolution, and a 3- to 5 -fold increase in photon sensitivity when compared to S-SPECT. ${ }^{3}$
DNM images were nondiagnostic in 4 (two men) of 168 patients $(2.4 \%)$ because of severe truncation artifact. These patients had large body habitus (BMI $\geq 40 \mathrm{~kg} / \mathrm{m}^{2}$ ), therefore it was more difficult to position their LV inside the camera quality field-of-view. Subsequent to this trial we have used new positioning software and increased technologist training and have been able to position all patients including patients as heavy as $358 \mathrm{lbs}(\mathrm{BMI}=$ 50) inside the quality field-of-view, thus avoiding the truncation artifacts. Moreover, the study population included 70 patients with $\mathrm{BMI}>30$, including 25 patients with BMI $>35$, in whom DNM images were judged of adequate or excellent quality.

There was statistically significant correlation of the left ventricular volumes and ejection fractions between DNM and S-SPECT. These results demonstrate that despite the higher contrast of the LV cavity in the DNM images as compared to S-SPECT images, ECTb was insensitive to these differences in measuring parameters of left ventricular function except for a small non-significant difference in resting end-diastolic volume. This is in part due to the border modeling approach (rather than border detection) used in $\mathrm{ECTb}^{15}$ and may not reflect results by other quantitative approaches.

There were high interobserver agreement rates for DNM images. We believe this is in part due to a consensus meeting among the three readers to evaluate 30 initial DNM imaging studies not included in the analysis prior to beginning the multicenter study.

Differences in breast tissue attenuation patterns have been noted between DNM and S-SPECT images, particularly in patients where the left breast attenuates only a portion of the left ventricle on rotational planar images of S-SPECT. These patients demonstrated relative count loss in the anterior wall on S-SPECT while there was none-to-minimal count loss in the anterior wall on DNM. Instead, some of these patients had a mild relative count loss in the inferior wall. We believe this is due to differences in detector geometry. The DNM system increases angular sampling by adding views in the longitudinal direction in contrast to the cylindrical geometry approach used on S-SPECT cameras.

\section{STUDY LIMITATIONS}

In this study we did not compare the ability of DNM and S-SPECT to assess the severity or myocardial extent of perfusion defects and the primary analysis was based on visual assessment. Computer-based quantitative analysis was not used for image interpretation or correlation of DNM and S-SPECT images because robust normal files were not available for DNM due to lack of additional studies. Future studies will use software-assisted quantification of perfusion defects. 


\section{PERSPECTIVES}

The ability to perform fast SPECT in list mode with stationary high sensitivity solid-state-detectors with multiple pinhole collimators greatly expands the opportunities in nuclear cardiology. It will provide better image quality at multiple energy levels, will allow fast imaging times and more efficient work flow, and will increase patient comfort with greater flexibility on imaging protocols. Moreover, this new technology facilitates the acquisition of true dynamic SPECT flow studies that may be used for measuring flow and myocardial flow reserve as well as for performing multi-compartmental modeling of radiopharmaceuticals.

\section{CONCLUSION}

A novel solid-state-detector dedicated cardiac camera was evaluated in this multicenter study. The new camera provided rest/stress Tc- $99 \mathrm{~m}$ tetrofosmin myocardial perfusion SPECT images comparable to conventional dual detector SPECT at significantly shorter acquisition times and with improved image quality.

\section{Acknowledgments}

This work was funded in part by GE Healthcare. Two of the authors $(R F, E V G)$ and Emory University receive royalties from the sale of the Emory Cardiac Toolbox ${ }^{T M}$ utilized to interpret images in the research described in this article. The terms of this arrangement have been reviewed and approved by Emory University in accordance with its conflict-of-interest practice.

\section{Open Access}

This article is distributed under the terms of the Creative Commons Attribution Noncommercial License which permits any noncommercial use, distribution, and reproduction in any medium, provided the original author(s) and source are credited.

\section{References}

1. Borges-Neto S, Pagnanelli RA, Shaw LK, et al. Clinical results of a novel wide beam reconstruction method for shortening scan time of Tc-99m cardiac SPECT perfusion studies. J Nucl Cardiol 2007; 14:555-65.
2. DePuey EG, Gadiraju R, Clark J, Thompson L, Anstett F, Shwartz SC. Ordered subset expectation maximization and wide beam reconstruction "half-time" gated myocardial perfusion SPECT functional imaging: A comparison to "full-time" filtered backprojection. J Nucl Cardiol 2008;15:547-63.

3. Garcia EV, Tsukerman L, Keidar Z. A new solid state ultra fast cardiac multi-detector SPECT System. J Nucl Cardiol 2008;15:S3 (abstract).

4. Hansen CL, Goldstein RA. Myocardial perfusion and function: Single photon emission computed tomography. ASNC Guidelines for Nuclear Cardiology Procedures. J Nucl Cardiol 2007;14:e3960.

5. Dunn OJ. Basic statistics: A primer for the biomedical sciences. New York: Wiley; 1977. p. 116-9.

6. Funk T, Despres P, Barber WC, Shah KS, Hasegawa BH. A multipinhole small animal SPECT system with submillimeter spatial resolution. Med Phys 2006;33:1259-68.

7. Beekman FJ, Vastenhouw B. Design and simulation of a highresolution stationary SPECT system for small animals. Phys Med Biol 2004;49:4579-92.

8. Jaszczak RJ, Li J, Wang H, Zalutsky MR, Coleman RE. Pinhole collimation for ultra-high-resolution, small-field-of-view SPECT. Phys Med Biol 1994;39:425-37.

9. Steele PP, Kirch DL, Koss JE. Comparison of simultaneous dualisotope multipinhole SPECT with rotational SPECT in a group of patients with coronary artery disease. J Nucl Med 2008;49:1080-9.

10. Volokh L, Hugg J, Blevis I, Asma E, Jansen F, Manjeshwar R. Effect of detector energy response on image quality of myocardial perfusion SPECT. Nuclear science symposium conference record. IEEE; 2008. ISSN:1082-3654, p. 4043-6.

11. Sharir T, Ben-Haim S, Merzon K, Prochorov V, Dickman D, Berman DS. High-speed myocardial perfusion imaging initial clinical comparison with conventional dual detector anger camera imaging. JACC Cardiovasc Imaging 2008;1:156-63; discussion 164-156.

12. Gambhir SS, Berman DS, Ziffer J, Naglr M, Sandler M, Patton J, et al. A novel high-sensitivity rapid-acquisition single-photon cardiac imaging camera. J Nucl Med 2009;50:635-43.

13. Berman DS, Kang X, Tamarappoo B, Wolak A, Hayes SW, Nakazato R, et al. Stress thallium-201/rest technetium-99m sequential dual isotope high-speed myocardial perfusion imaging. JACC Cardiovasc Imaging 2009;2:273-82.

14. Volokh L, Lahat C, Binyamin E, Blevis I. Myocardial perfusion imaging with an ultra-fast cardiac SPECT camera-a phantom study. Nuclear science symposium conference record. NSS ' 08 . IEEE; 2008. ISSN: 1082-3654, p. 4636-9.

15. Faber TL, Cooke CD, Folks RD, Vansant JP, Nichols KJ, DePuey $\mathrm{EG}$, et al. Left ventricular function and perfusion from gated SPECT perfusion images: An integrated method. J Nucl Med 1999;40:650-9. 